\title{
Clinical Evaluation of Hypothalamo-Pituitary-Thyroid Axis Hormones in Critically Ill Children. Cross Sectional Study at Zagazig University Children Hospital Mohamed Nageeb Abo Elfottoh ${ }^{1}$, Hossam Mostafa Kamal ${ }^{1}$, Ahmed Mohamed Gab-allah ${ }^{2}$, Eman El-Sayed Ali*1 \\ Departments of ${ }^{1}$ Pediatric and ${ }^{2}$ Clinical Pathology, Faculty of Medicine - Zagazig University \\ *Corresponding author: Eman El-Sayed Ali, Mobile: (+20)01280997732, Email: doctor.eman.deee@ gmail.com
}

\begin{abstract}
Background: Critical illness is the medical condition in which a patient requires immediate intensive medical support of vital organ functions in order to survive. Independent of the underlying condition, critical illness is characterized by a uniform dysregulation of the hypothalamic-pituitary-peripheral axes. In the majority of these axes a clear biphasic pattern can be distinguished.

Objective: To evaluate serum concentration of adrenocorticotrophin hormone, cortisol, TSH, free T4 in children who had severe illness in Pediatric Intensive Care Unit at Zagazig University Hospitals and El-Ahrar Hospital in Pediatric Intensive Care Unit.

Patients and Methods: This study was cross sectional study which conducted at Zagazig University Hospitals and El-Ahrar Hospital in Pediatric Intensive Care Unit on 30 critically ill children. These investigations were measured for all participating patients (serum level of TSH, free T4, adrenocorticotrophin hormone and cortisol).

Results: This study was conducted on 30 critically ill children. Mean \pm SD of age was $12.9 \pm 18.0$ years. $53 \%$ were males. Serum cortisol level was high in about $43 \%$ of our critically ill children patients. Serum concentration of adrenocorticotrophin hormone was lower in about $90 \%$ in children who had severe illness in pediatric intensive care unit. Serum concentration of TSH was high in about $23 \%$ of our critically ill children patients. Serum concentration of free T4 in children was high in about $20 \%$ while was lower in $6.7 \%$ of our critically ill children patients. Secondary thyroid insufficiency represented $7 \%$ (2/30 cases).

Conclusion: Secondary thyroid insufficiency represented in $7 \%$ of our critically ill children patients. No statistically significant differences in baseline characteristics of the studied critically ill children (stratified according to cortisol level). No statistically significant differences in ACTH, TSH and free T4 levels of the studied critically ill children (stratified according to cortisol level).
\end{abstract}

Keywords: Critically Ill Children, Hypothalamo-Pituitary-Thyroid Axis Hormones

\section{INTRODUCTION}

Critical illness is the medical condition in which a patient, because of major surgery or severe illness, requires immediate intensive medical support of vital organ functions in order to survive. Independent of the underlying condition, critical illness is characterized by a uniform dysregulation of the hypothalamic-pituitaryperipheral axes. In the majority of these axes a clear biphasic pattern can be distinguished ${ }^{(\mathbf{1})}$.

The metabolic responses to critical illness involve every organ and tissue of the body and yet, surprisingly, little is known about the underlying mechanisms. During critical illnesses, the state of stress results in hypermetabolism, increased energy expenditure, hyperglycemia and muscle loss (2).

The thyroid axis, at the level of the hypothalamus, thyrotropin-releasing hormone (TRH) is released and stimulates the thyrotropic cells in the pituitary to synthesize and secrete thyroid-stimulating hormone (TSH). TSH, secreted in a pulsatile and diurnal pattern, in turn drives the thyroid gland to synthesize and secrete thyroid hormones. Euthyroid Sick Syndrome (ESS) is the commonest endocrine change seen in critically ill patients. It is described as abnormalities in circulating thyroid hormone levels without pre-existing hypothalamic pituitary or thyroid gland dysfunction in the setting of a Non-Thyroidal Illness (NTI) ${ }^{(3)}$.

Critical illness also leads to a cascade of physiological changes. In particular, the activation of the hypothalamic-pituitary-adrenal (HPA) axis brings about changes necessary for an adequate stress response. High cortisol levels was reported during critical illness in both adults and children, although there is considerable individual variation ${ }^{(4)}$.

Activation of the HPA axis is necessary for everyday functioning, but excessive acute stimulation, such as that experienced during critical illness may play a role in the development of subsequent psychological problems. HPA axis dysregulation was connected with adverse childhood experiences involving maltreatment and with a number of pediatric psychological problems including anxiety and post-traumatic stress disorder (PTSD) ${ }^{(2)}$.

Abnormalities in thyroid function tests in patients with non-thyroidal illness may be divided into low T3 syndrome, low T3 and low T4 syndrome, high T4 syndrome and a mixed form. Hence it is becoming clear that the conversion of $\mathrm{T} 4$ to $\mathrm{T} 3$ can

This article is an open access article distributed under the terms and conditions of the Creative Commons Attribution (CC BY-SA) license (http://creativecommons.org/licenses/by/4.0/) 
vary according to the clinical state, which can therefore influence the metabolic action of thyroid hormone and the function of hypothalamo-pituitarythyroid axis. The severity of these neuroendocrine alterations was shown to be related to adverse outcome of patients in the intensive care unit ${ }^{(5)}$.

In children with septic shock and meningococcal disease, both high and low cortisol levels were associated with increased illness severity and poor physical outcomes. Inadequate cortisol levels in the face of severe physiological stress and associations between cortisol and illness severity were also reported in other critical illnesses ${ }^{(6)}$. The aim of this work were to evaluate serum concentration of adrenocorticotrophin hormone, cortisol, TSH, free T4 in children who had severe illness in Pediatric Intensive Care Unit at Zagazig University Hospitals and El-Ahrar Hospital in Pediatric Intensive Care Unit.

\section{SUBJECTS AND METHODS}

Site of the Study: This study was cross sectional study, which was conducted at Zagazig University Hospitals El-Ahrar Hospital in Pediatric Intensive Care Unit.

Sample size: Assuming that rate of admission of critically ill children in children hospital Zagazig University is 5 cases/month. So, a comprehensive sample of 30 children was included in the study.

\section{Inclusion criteria:}

- All children with age range from 1 month to 6 years who were admitted to pediatric intensive care unit and were in a critical condition.

- Children with severe illness (respiratory distress, polytrauma, endocrinal disturbance, circulatory disturbance, central nervous system insult).

\section{Exclusion criteria:}

1. Absence of informed consent.

2. Age group out of range (1 month to 6 years).

3. Children not admitted to pediatric intensive care unit.

\section{All the participating patients were subjected to the following:}

These investigations were measured (serum level of TSH, free T4, adrenocorticotrophin hormone and cortisol). Hormonal Assay for determination of (ACTH, Cortisol, TSH and Free T4).

\section{Technique:}

Sampling: $4 \mathrm{ml}$ from venous blood were collected under complete aseptic conditions from all patients at 9 a.m., were divided into $2 \mathrm{ml}$ in plain tube for determination of TSH, free T4 and cortisol and $2 \mathrm{ml}$ on K3 EDITA for determination of ACTH. Samples were immediately centrifugated at $3000 \mathrm{r}$ p.m. for 10 minutes at room temperature. Serum and plasma were separated and immediately the analytes were measured.

Principle: By quantitative determination in human serum by the electrochemical luminescence competitive immunoassay (ECLIA) used on Cobas (England) with immunoassay analyser E 411 Roche Diagnostics.

\section{Ethical approval:}

Approval was taken from the Institutional Review Board (IRB) of Faculty of Medicine Zagazig University. Informed consent was obtained from written informed consent was taken from parents for participation in the study.

\section{Statistical analysis}

Data were analyzed by Statistical Package of the Social Sciences (SPSS), software version 24. Continuous data were presented as mean $\pm S D$ if normally distributed or median (range) if not normally distributed. Categorical data were presented as frequency and percentage. Normality was checked by Kolmogorov-Smirnov test. Kruskal-Wallis H test was used to compare more than 2 groups and Mann-Whitney $\mathrm{u}$ test was used to compare 2 groups. Pearson's correlation coefficient ( $\mathrm{r}$ ) was calculated. $\mathrm{P}$-value $<0.05$ indicated significance. Two-sided tests were used throughout.

\section{RESULTS}

This study was conducted 30 critically ill children. Mean \pm SD of age was $12.9 \pm 18.0$ years. $53 \%$ was males (Table 1).

Table (1): Baseline characteristics of the studied critically ill children.

\begin{tabular}{|lc|}
\hline Variables & $\boldsymbol{n}=\mathbf{3 0}$ \\
\hline Age (months) & \\
Mean \pm SD & $12.9 \pm 18.0$ \\
Median (range) & $6(2-72)$ \\
\hline Sex, $\boldsymbol{n},(\boldsymbol{\%})$ & \\
Male & $16(53)$ \\
Female & $14(47)$ \\
\hline * Associated critical & \\
illness, $\boldsymbol{n},(\boldsymbol{\%})$ & \\
$\dagger$ Respiratory illness & $13(43)$ \\
†Neurologic illness & $7(23)$ \\
Severe dehydration & $5(17)$ \\
Heart failure & $2(7)$ \\
$\|$ Miscellaneous & $3(10)$ \\
\hline *Most prominent diagnosis \\
$\dagger \dagger$ includes pneumonia, asthmatic bronchitis \\
† includes spinal muscle atrophy, hemorrhage, \\
encephalitis \\
$\|$ includes polytrauma, intestinal obstruction, \\
hemolytic uremic syndrome \\
\hline
\end{tabular}

Serum cortisol level was high in about $43 \%$ of our critically ill children patients. Serum concentration of adrenocorticotrophin hormone was lower in about $90 \%$ in children who had severe illness in Pediatric Intensive Care Unit. Serum concentration of TSH was high in about $23 \%$ of our critically ill children patients. Serum concentration of free $\mathrm{T} 4$ in children was high in about $20 \%$ while was lower in $6.7 \%$ of our critically ill children patients (Figure 1). 


\section{DISCUSSION}

In our study, no statistically significant correlation between ACTH level and age of the studied critically ill children was found. Similarly, no statistically significant differences in sex, associated complications. There was no statistically significant correlations between ACTH level and laboratory findings of the studied critically ill children, but ACTH level was significantly lower in non-survivors compared to survivors.

This agrees with Boonen et al. ${ }^{(7)}$ who found that, that ACTH levels are often only transiently increased or not increased at all in critical illness.

In contrary to the current study, Cuhaci et al. ${ }^{(8)}$ who evaluated the pituitary-adrenal-gonadal-thyroid axis in the Intensive Care Unit (ICU) patients, their outcome, the association between these hormonal changes. They found no statistically significant difference between non-survivors compared to survivors regarding ACTH level.

Menon et al. ${ }^{(9)}$ reported that the prevalence of adrenal insufficiency was $30.2 \%$ in the critically ill neonates.

Also, the findings Quintos and Boney ${ }^{(10)}$ in their review article, they demonstrated that numerous ill newborns have improperly low serum cortisol concentrations. Adrenal insufficiency is transient and likely reflects normal adrenal physiology at younger gestational ages.

No statistically significant correlation between cortisol level and age of the studied critically ill children was found. Similarly, no statistically significant differences in sex, associated complications and outcome were observed. No statistically significant correlations between cortisol level and laboratory findings of the studied critically ill children was found.

This disagrees with Ray et al. (11) who found cortisol concentration was higher in non-survivors.

Levy-Shraga and Pinhas-Hamiel ${ }^{(12)}$ found that many critically ill patients have 'relative' or 'functional' adrenal insufficiency, which is characterized by an inadequate production of cortisol in relation to an increased need throughout times of severe stress. However, most critically-ill patients have elevated plasma cortisol concentrations, which reflect activation of the hypothalamic-pituitary-adrenal axis as a homeostatic adaptation.

Karlsson et al. ${ }^{(13)}$ showed that higher cortisol baseline was observed in non-survivors septic patients, as observed in large cohort studies.

Pont-Thibodeau et al. ${ }^{(14)}$ informed that increased mortality rate is linked to both high and low cortisol levels. Moreover, clinical studies show that patients with relative adrenal insufficiency are at a significantly higher risk of hospital mortality ${ }^{(15)}$.
In the current work, no statistically significant correlation between free T4 level and age of the studied critically ill children was found. Similarly, no statistically significant differences in sex, associated complications and outcome were observed. No statistically significant correlations between TSH level and laboratory findings of the studied critically ill children. A statistically significant positive correlation between TSH level and age of the studied critically ill children was found, but no statistically significant differences in sex, associated complications and outcome were observed. No statistically significant correlations between TSH level and laboratory findings of the studied critically ill children.

This disagrees with Ray $\boldsymbol{e t}$ al. ${ }^{(11)}$, who compared thyroid and adrenal function between survivors and non-survivors in critical illness. They concluded that thyroid hormone concentration was significantly lower in non-survivors than in survivors.

Bhat et al. ${ }^{(16)}$ measured free tetraiodothyronine (fT4) and TSH (Thyroid Stimulating Hormone) levels in critically ill patients. They found that $59 \%$ of the critically ill patients admitted in wards and ICUs showed abnormality in one or more than one parameters of the thyroid profile. High TSH and low fT4 levels were common abnormalities. Out of all the critically ill patients screened for thyroid disorders, $73.2 \%$ were found to be females. This preponderance of the female patients in the present study might be attributed to the fact that thyroid disorders being more common in females are more likely to raise physician's suspicion. This may be the reason for high screening rate in the female patients. Moreover, hormonal differences between the two genders, working traditions, body weight, meal schedules, dietary factors or ingestion of drugs among other factors may be responsible for the observed gender differentiation in their study. Furthermore, it was found that in critically ill patients, low fT3 (50\%) was the commonest abnormality followed by high TSH (12.4\%), low TSH $(8.8 \%)$ and low fT4 (4.7\%) concentration.

\section{CONCLUSION}

Serum cortisol level was high in about $43 \%$ of our critically ill children patients. Serum concentration of adrenocorticotrophin hormone was lower in about 90\% in children who had severe illness in pediatric intensive care unit. Serum concentration of TSH was high in about $23 \%$ of our critically ill children patients. Serum concentration of free T4 in children was high in about $20 \%$ while it was lower in $6.7 \%$ of our critically ill children patients. No adrenal insufficiency (peripheral and central) was founded in our study. Secondary thyroid insufficiency represented in $7 \%$ of our critically ill children patients. No statistically significant differences in baseline characteristics of the studied critically ill children was found (stratified 
according to cortisol level). No statistically significant differences in ACTH, TSH and free T4 levels of the studied critically ill children (stratified according to cortisol level) were found.

\section{REFERENCES}

1. Als L, Picouto M, Hau S et al. (2015): Mental and physical well-being following admission to paediatric intensive care. Pediatr Crit Care Med., 16:141-149.

2. Hollocks M, Pickles A, Howlin P et al. (2016): Dual Cognitive and Biological Correlates of Anxiety in Autism Spectrum Disorders. J Autism Dev Disord., 46(10): 3295307.

3. Haas N, Camphausen C, Kececioglu D (2006): Clinical review: Thyroid hormone replacement in children after cardiac surgery: is it worth a try? Crit Care, 10: 213-6.

4. Goodman S, Sprung C, Ziegler D et al. (2005): Cortisol changes among patients with septic shock and the relationship to ICU and hospital stay. Intensive Care Med., 31:1362-1369.

5. Peeters R, Wouters $P$, van Toor $H$ et al. (2005): Serum 3,3',5'-triiodothyronine (rT3) and 3,5,3'triiodothyronine/rT3 are prognostic markers in critically ill patients and are associated with postmortem tissue deiodinase activities. J Clin Endocrinol Metab., 90:45594565 .

6. Küçükemre B, Demirkol D, Bas F et al. (2014): Evaluation of endocrine function in children admitted to pediatric intensive care unit. Pediatr Int., 56:349-353

7. Boonen $E$, Vervenne $H$, Meersseman $P$ et al. (2013): Reduced cortisol metabolism during critical illness. N Engl J Med., 368:1477- 1488.
8. Cuhaci N, Ogmen B, Doger C (2017): Association of hormonal changes with disease severity and mortality rate in critically ill patients. Turk J Endocrinol Metab., 21:1-8

9. Menon K, Ward R, Lawson $M$ et al. (2010): A Prospective multicenter study of adrenal function in critically ill children. Am J Respir Crit Care Med., 182: 246-251.

10. Quintos J, Boney C (2010): Transient adrenal insufficiency in the premature newborn. Curr Opin Endocrinol Diabetes Obes., 17: 8-12.

11. Ray D, Macduff A, Drummond G et al. (2002): Endocrine measurements in survivors and non-survivors from critical illness. Intensive Care Med., 28:1301-1308.

12. Levy-Shraga Y, Pinhas-Hamiel O (2013): Critical illness-related corticosteroid insufficiency in children. Horm Res Paediatr., 80:309-317.

13. Karlsson S, Varpula M, Ruokonen E et al. (2007): Incidence, treatment, and outcome of severe sepsis in ICU-treated adults in Finland: The Finnsepsis study. Intensive Care Med., 33: 435-443.

14. Pont-Thibodeau D, Joyal J, Lacroix J (2014): Management of neonatal sepsis in term newborns. F1000Prime Rep., 6: 67-73.

15. Annane D, Sebille V, Troche G et al. (2000): A 3-level prognostic classification in septic shock based on cortisol levels and cortisol response to corticotropin. JAMA., 283:1038-45.

16. Bhat K, Sharma S, Sharma K et al. (2016): Assessment of thyroid function in critically ill patients. Biomedical Research, 27 (2): 449-452. 